DOI: http://dx.doi.org/10.5007/2175-8069.2016v13n29p113

\title{
Emprego de métodos multicritério em decisões gerenciais: uma análise bibliométrica da produção científica brasileira
}

\author{
Use of MultiCriteria methods in management decisions: bibliometric analysis of brazilian \\ scientific production
}

Empleo de métodos multicriterio en decisiones gerenciales: un análisis bibliométrico de la producción científica brasileña

\author{
André Andrade Longaray \\ Doutor em Engenharia de Produção na Universidade Federal de Santa Catarina \\ Professor do Mestrado em Administração da Universidade Federal do Rio Grande - FURG \\ Endereço: Av. Itália, km 8, s/n, Campus Carreiros \\ CEP: 96.201-900 - Rio Grande/RS - Brasil \\ E-mail: longaray@yahoo.com.br \\ Telefone: + 55 (53) 3293-5081 \\ Vilmar Antonio Gonçalves Tondolo \\ Doutor em Administração na Universidade do Vale do Rio dos Sinos - UNISINOS \\ Professor do Mestrado em Administração da Universidade Federal do Rio Grande - FURG \\ Endereço: Av. Itália, km 8, s/n, Campus Carreiros \\ CEP: 96.201-900 - Rio Grande/RS - Brasil \\ E-mail: vtondolo@gmail.com \\ Telefone: + 55 (53) 3293-5081
}

Paulo Roberto Munhoz

Especialista em Tecnologia Educacional na Universidade Federal do Rio Grande - FURG

Professor do Instituto de Ciências Humanas e da Informação - ICHI/FURG

Endereço: Av. Itália, km 8, s/n, Campus Carreiros

CEP: 96.201-900 - Rio Grande/RS - Brasil

E-mail:paulorsmunhoz@hotmail.com

Telefone: + 55 (53) 3293-5081

\section{Rosana da Rosa Portella Tondolo}

Doutora em Administração na Universidade do Vale do Rio dos Sinos - UNISINOS

Professora do Mestrado em Desenvolvimento Territorial e Sistemas Agroindustriais da

Universidade Federal de Pelotas - UFPEL

Endereço: Rua Gomes Carneiro, n॰ 1, sala 410, Centro

CEP: 96.010-610 - Pelotas/RS - Brasil

E-mail: rosanatondolo@gmail.com

Telefone: + 55 (53) 3921-1326

Artigo recebido em 01/03/2016. Revisado por pares em 07/04/2016. Reformulado em 15/05/2016. Recomendado para publicação em 01/07/2016 por Sandra Rolim Ensslin (Editora Científica). Publicado em 26/08/2016. 


\title{
Resumo
}

O processo decisório possui papel essencial em uma organização, e a pesquisa operacional, como ramo da ciência dedicado à decisão, oferece uma diversidade de métodos para gestores avaliarem suas alternativas. Dentre as possibilidades, os métodos multicritério têm sido amplamente empregados em contextos decisórios envolvendo as áreas contábil, administrativa e de operações das organizações. A presente pesquisa descreve a análise da produção científica brasileira sobre a utilização de métodos multicritério aplicados no âmbito das decisões gerenciais. Para atender ao objetivo proposto foi desenvolvida uma análise bibliométrica dos periódicos e anais de eventos das áreas de Ciências Contábeis, Administração e Engenharia de Produção, levando em conta a adequação da linha temática e editorial. O período de abrangência da pesquisa foi o transcorrido de 2004 até 2016. De uma amostra inicial de 1.087 artigos, obteve-se um Portfólio Bibliográfico de 623 artigos. Identificaram-se os métodos $M A C B E T H$ e $A H P$ como mais empregados, e a existência de redes de pesquisadores na área multicritério.

Palavras-chave: Métodos multicritério. Decisões gerenciais. Bibliometria.

\begin{abstract}
The decision-making process has an important role in an organization. The Operations Research, as a branch of science devoted to the decision, offers a variety of methods for managers to evaluate their alternatives. Among the possibilities, the MultiCriteria methods have been widely used in decisionmaking contexts involving the accounting, administrative and operations organizations. This research describes the analysis of Brazilian scientific literature on the use of MultiCriteria methods applied in the context of management decisions. In order to meet the proposed objective a bibliometric analysis of journals and annals of events in the areas of accounting, administration and production engineering was developed, taking into account the adequacy of thematic and editorial line. The scope of the research period has elapsed from the year 2004 until the year 2016. From an initial sample of 1,087 articles, we obtained a bibliographic portfolio of 623 articles. We identified the MACBETH and AHP methods as more employees, and the existence of networks of researchers in MultiCriteria area.
\end{abstract}

Keywords: MultiCriteria methods. Managerial decision. Bibliometric research.

\section{Resumen}

El proceso de decisión posee papel esencial en una organización, y la investigación operacional como rama de la ciencia dedicada para decisión ofrece una diversidad de métodos para que gestores puedan evaluar sus alternativas. Entre las posibilidades, los métodos multicrierio han sido ampliamente empleados en contextos de decisión, involucrando las áreas contable, administrativa y de operaciones de las organizaciones. La presente investigación describe el análisis da producción científica brasileña sobre el uso de métodos multicriterio aplicados en el ámbito de las decisiones gerenciales. Para atender al objetivo propuesto fue desarrollado un análisis bibliométrico de las revistas científicas y anales de eventos de las áreas de Ciencias Contables, Administración e Ingeniería de Producción, considerando la adecuación de la línea temática y editorial. El período de cobertura de la investigación fue lo trascurrido de 2004 hasta 2016. De una muestra inicial de 1.087 artículos se obtuve un Portfolio Bibliográfico de 623 artículos. Se identificaron los métodos MACBETH y AHP como los más empleados, y la existencia de redes de investigadores en el área multicriterio.

Palavras clave: Métodos multicriterio. Decisiones gerenciales. Bibliometría. 


\section{Introdução}

No dia a dia, a gestão organizacional se depara com inúmeras situações nas quais é impelida a tomar decisões. Mesmo sem perceber, decisões são tomadas a todo momento. $\mathrm{O}$ processo de tomada de decisão é inerente a qualquer atividade desenvolvida pelo homem e, no ambiente organizacional, não é diferente. Os gestores, para assegurarem o desenvolvimento das organizações, têm que tomar os mais variados tipos de decisões a fim de atingir os objetivos e as metas organizacionais.

Entretanto, com a crescente concorrência, com o aumento das exigências do mercado e com o ritmo frenético das mudanças, o processo de tomada de decisão se torna cada vez mais complexo, pois envolve um grande número de variáveis, deixando as melhores alternativas cada vez menos evidentes.

Uma das abordagens utilizadas para auxiliar os gestores na difícil tarefa de tomar decisões, nesse ambiente complexo e dinâmico, são os métodos multicritério da pesquisa operacional. Eles visam tornar os problemas mais claros, facilitando a avaliação das alternativas disponíveis frente à multiplicidade de critérios existentes em cenários complexos. Ao aplicar tais técnicas, os problemas tornam-se mais estruturados e robustos, facilitando a avaliação das alternativas disponíveis.

Diante disso, percebe-se que os métodos multicritério de apoio à decisão podem se configurar em instrumentos úteis para o gestor organizacional atualmente. Assim, o conhecimento da literatura sobre o assunto ganha importância para orientar esses gestores a respeito das melhores práticas, bem como pode balizar pesquisadores em estudos futuros.

Tendo em vista o panorama exposto, surge a questão que norteou a presente pesquisa: Como se caracteriza a produção científica brasileira sobre a aplicação de métodos multicritério aplicados a contextos gerenciais de tomada de decisão?.

Nessa direção, o objetivo do presente estudo é analisar a produção científica brasileira sobre a aplicação de métodos multicritério em processos decisórios no âmbito do contexto gerencial, por meio de uma análise bibliométrica de artigos publicados no período compreendido entre 2004 e 2016.

A fim de atingir o objetivo geral, este estudo teve como delineadores os seguintes objetivos específicos: (i) Levantar o número de artigos publicados ao ano; (ii) Destacar o número de autores por artigo; (iii) Listar os autores mais prolíferos; (iv) Revelar a participação das universidades; (v) Elencar os métodos multicritério mais aplicados; (vi) Identificar as áreas de aplicação; (vii) Arrolar as obras de maior repercussão entre os artigos; (viii) Divulgar os artigos e livros de maior relevância; e (ix) Evidenciar artigos e autores de destaque.

O presente artigo divide-se em cinco seções. Decorrida a introdução, a seção 2 apresenta o marco teórico referente aos métodos multicritério da pesquisa operacional. Após, na seção 3, realiza-se o enquadramento metodológico da pesquisa servindo como base ao detalhamento do levantamento bibliométrico realizado no estudo (seção 4). Por fim, a seção 5 descreve as considerações finais acerca da pesquisa, apresentando também as limitações do presente trabalho e sugestões para futuros estudos de temática semelhante. Após, apresentamse as referências bibliográficas. 


\section{Métodos Multicritério}

A utilização de métodos multicritério para auxiliar os processos de tomada de decisão tem crescido nos últimos anos. Prova disso são os trabalhos publicados em periódicos internacionais de vários segmentos. Entre eles, pode-se destacar: European Journal of Operational Research - EJOR; Journal of Operations Research Society - JORS; Decision Science-DS; e Management Decision - MD.

No Brasil, a mesma tendência tem se replicado. Há mais de 20 anos, ano após ano a aplicação de métodos multicritério é assunto constante em encontros, congressos e simpósios, como pode ser verificado nos anais dos Congressos da Associação Nacional dos Programas de Pós-Graduação em Contabilidade - ANPCONT, Congresso Brasileiro de Custos - CBC, Encontro Nacional dos Programas de Pós-graduação em Administração - ENANPAD, Encontro Nacional de Engenharia de Produção - ENEGEP e Simpósio Brasileiro de Pesquisa Operacional - SBPO, dentre outros.

Além disso, revistas como Revista Contemporânea de Contabilidade - RCC, Revista Contabilidade e Finanças, Revista $\mathrm{ABC}$ Custos, Revista de Educação e Pesquisa em Contabilidade, Revista Produção, Revista Produção Online, Revista Pesquisa Operacional e Gestão Industrial, por exemplo, têm publicado novos artigos sobre o assunto (ALBUQUERQUE; 2011, BORTOLUZZI; SILVA; ENSSLIN; ENSSLIN; 2013, ENSSLIN; DUTRA; MARTINS; DEZEM, 2016).

Sob essa perspectiva, Belton e Stewart (2002) definem os métodos multicritério como técnicas científicas que oferecem suporte ao processo de tomada de decisão que possui uma diversidade de indicadores em situações complexas. Sinteticamente, a operacionalização do uso de um método multicritério para avaliar possibilidades em uma determinada decisão ocorre pela decomposição dos objetivos em critérios, que recebem valores de importância e têm alternativas de decisão avaliadas em cada um dos critérios estabelecidos. Construído o modelo, pode-se realizar a avaliação global de qualquer alternativa proposta, levando em conta o peso de cada critério e sua performance no modelo desenvolvido (KEENEY; RAIFFA, 1976).

Matematicamente, os métodos multicritério podem ser divididos em dois ramos. O primeiro é o contínuo, denominado Multiobjective Decision Making (MODM), orientado a problemas com objetivos múltiplos, nos quais as alternativas podem adquirir um número infinito de valores. O segundo ramo é o discreto, nominado Multi Attribute Decision Making $(M A D M)$, que analisa problemas nos quais o conjunto de alternativas de decisão é formado por um número finito e previsível de variáveis (TRIANTAPHYLLOU; SHU; NIETO SANCHEZ; RAY, 1998).

Há uma diversidade de métodos de multicritérios que podem ser encontrados na literatura (ENSSLIN; MONTIBELLER; NORONHA; 2001): AHP - Analytic Hierarchical Process, desenvolvido por Thomas Saaty (1990); MACBETH - Measuring Attractiveness by a Categorical Based Evaluation Technique, desenvolvido por Bana E Costa e Vansnick (1995); TOPSIS - Technique for Order Preference by Smilarity to Ideal Solution, desenvolvido por Hwang, Lai and Liu (1993); Família ELECTRE - ELimination Et Choice TRaidusaint la Realitè (ROY, 1991); PROMETHEE - Preference Ranking Organization METHod for Enrichment of Evaluations (BRANS, 1982); dentre outros.

Embora os métodos guardem entre si diferenças sob a perspectiva matemática ou de nomenclatura, todos são empregados para o mesmo fim: o desenvolvimento de modelos ou 
instrumentos para auxiliar a tomada de decisão, baseados no uso de métodos multicritério (BRANS; VINCKE; MARESCHAL, 1986; VINCKE, 1989; ROY, 1991; ROY; BOUYSSOU, 1993; SCHÄRLIG, 1996; BARBA-ROMERO; POMEROL, 1997; ENSSLIN; MONTIBELLER; NORONHA, 2001, BANA E COSTA; DE CORTE; VANSNICK, 2005, RANGEL; GOMES, 2010).

\section{Procedimentos Metodológicos}

A fim de explicitar os procedimentos adotados nesta pesquisa, esta seção está organizada em enquadramento metodológico geral, procedimentos de coleta de dados e procedimentos de análise de dados.

Este estudo se caracteriza como descritivo, ocorrendo a descrição das características dos artigos que compõem o Portfólio Bibliográfico. Como estratégia, empregou-se a pesquisa bibliográfica, a qual, segundo Gil (2010), é elaborada com base em materiais já publicados, tais como livros, revistas, jornais, teses, dissertações e anais de eventos científicos, caracterizando-se, assim, como fonte secundária de dados. Dessa forma, a pesquisa é predominantemente quantitativa, uma vez que converte as descrições das informações dos trabalhos analisados em dados para serem tratados estatisticamente.

A realização desta pesquisa consiste no procedimento de duas etapas da análise bibliométrica. Na primeira etapa, objetivou-se a coleta dos dados referentes ao propósito do estudo. Já na etapa seguinte, apresentam-se os resultados obtidos na análise. A etapa de coleta de dados, para formação do Portfólio Bibliográfico, consistiu da seleção e do exame de artigos disponibilizados em bases de dados online. Para a realização desta pesquisa foram utilizados, como fonte de dados, bancos de artigos online, provenientes de anais de encontros e simpósios, bem como de periódicos de destaque nacional nas áreas de Administração, Engenharia de Produção e Ciências Contábeis (Quadro 1). Assim, acredita-se que a amostra de artigos selecionada seja capaz de representar com fidelidade a produção científica brasileira sobre a aplicação de métodos multicritério.

\section{Quadro 1- Fontes de dados utilizadas na pesquisa}

\begin{tabular}{|ll|}
\hline ANAIS & \\
\hline EnADI & (Encontro de Administração da Informação) \\
EnANPAD & (Encontro da Associação Nacional de Pós-Graduação e Pesquisa em Administração) \\
ENEGEP & (Encontro Nacional de Engenharia de Produção) \\
SBPO & (Simpósio Brasileiro de Pesquisa Operacional) \\
SIMPEP & (Simpósio de Engenharia de Produção) \\
CBC & (Congresso Brasileiro de Custos) \\
ANPCONT & Congresso ANPCONT) \\
\hline PERIÓDICOS & \\
\hline Exacta - EP & \\
Gestão \& Produção \\
Pesquisa Operacional \\
PO para o Desenvolvimento (Pesquisa Operacional para o Desenvolvimento) \\
Produção \\
Produção Online \\
Produto \& Produção \\
Revista ABC Custos \\
Revista de Administração Contemporânea - RAC \\
Revista Contemporânea de Contabilidade - RCC \\
\hline
\end{tabular}




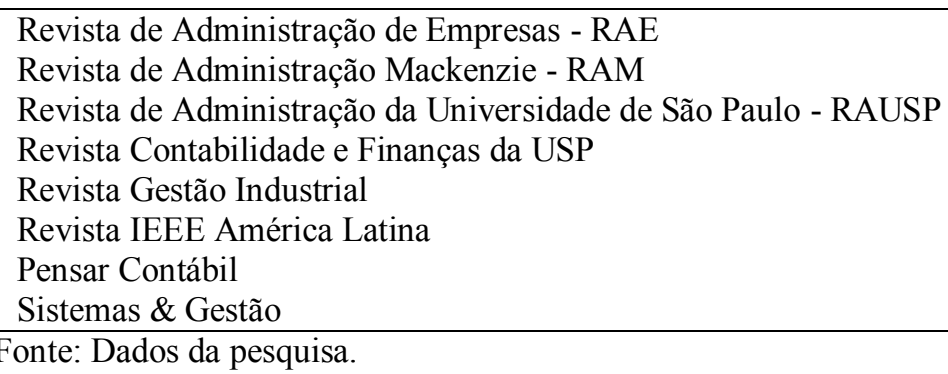

Uma vez definidas as fontes de dados para a pesquisa, foi executada a coleta dos artigos nos periódicos que constam no Quadro 1. Em geral, a busca teve como período de avaliação os artigos publicados entre 2004 e 2015 para os anais de congressos, e de 2004 até junho de 2016 para os periódicos da base. Tomando por referência Rodriguez, Costa e Carmo (2013), utilizou-se a seguinte expressão booleana: AHP OR ELECTRE OR Kepner OR MACBETH OR MAUT OR MAVF OR MAVT OR MCDA OR MCDM OR Multicritério OR PROMETHEE OR PROMSORT OR SMARTER OR SMARTS OR TODIM OR TOMASO OR TOPSIS OR UTADIS OR ZAPROS OR "Analytic Network Process" OR "Rating Technique" OR "Utilité Additive" OR "Variable Interdependent Parameters". Como pode ser observado, essa expressão contempla os principais métodos multicritério.

Nos casos em que não foi possível utilizar a expressão, múltiplas pesquisas foram executadas com cada uma das palavras-chave que a compõe, de forma a obter o mesmo resultado de sua utilização.

Em especial, para os anais do SBPO (de 2004 a 2015) e do SIMPEP (de 2007 a 2015), os quais apresentam seus artigos classificados em categorias, facilitando a identificação, foi realizado o download de todos os artigos da seção "MC: Multicritério" (de 2004 a 2015) e da seção "ADM: Apoio à Decisão Multicritério" (de 2010 a 2015) dos anais do SBPO e de todos os artigos da área "6.2 Decisão Multicriterial" (de 2007 a 2015) dos anais do SIMPEP.

Ao final do processo de coleta, obteve-se um total de 1.087 artigos, sendo que, destes, 464 não se alinhavam com o tema e foram descartados. Assim, foi possível selecionar, para a análise bibliométrica, 623 artigos ao total.

A técnica de análise de dados consistiu na análise bibliométrica que fundamenta o objetivo de pesquisa proposto. Esse método compõe-se de técnicas estatísticas e matemáticas para descrever aspectos da literatura e de outros meios de comunicação (ARAÚJO, 2006; VALMORBIDA; ENSSLIN, 2016). Para tal, a análise dos dados do Portfólio Bibliográfico seguiu os passos e técnicas baseando-se em Dutra et al. (2015), Ensslin et al. (2014) e Valmorbida e Ensslin (2016).

\section{Apresentação e Discussão dos Resultados}

Quanto à análise dos resultados, adotou-se a estrutura utilizada por Longaray et al. (2015), na qual se determinam categorias quanto ao fim da investigação. Assim, os dados foram divididos em (i) estudo da produção e autoria; (ii) estudo do conteúdo; e (iii) estudo das referências bibliográficas.

\subsection{Estudo da produção e autoria}


Para analisar a produção e autoria do Portfólio Bibliográfico levantou-se o número de publicações por ano. Cada artigo foi classificado em relação ao número de autores por artigo, e identificaram-se os autores $\mathrm{e}$ as universidades que mais contribuíram para o desenvolvimento do tema em pesquisa, por meio da determinação daqueles que possuem o maior volume de artigos dentre os selecionados. Assim, foi elaborado um histograma com o número de publicações dos artigos selecionados, agrupados por ano, com sua respectiva linha de tendência linear, como pode ser observado na Figura 1.

Figura 1 - Estudo das publicações

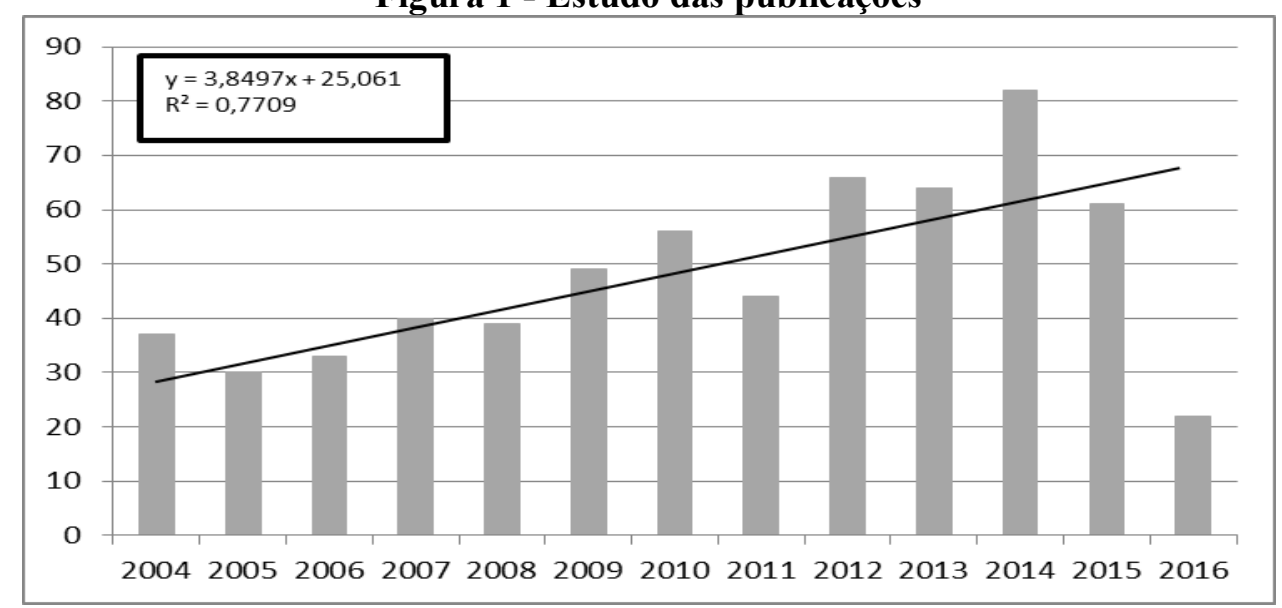

Fonte: Dados da pesquisa.

Dessa forma, verifica-se que o volume de produção vem assumindo uma tendência de crescimento, sendo esta de quatro a seis artigos por ano com coeficiente de determinação $\left(\mathrm{R}^{2}\right)$ de 0,77 , dentro do período analisado. Além disso, classificando os artigos em relação ao número de autores, nota-se que, no período analisado, a maior parte dos artigos é escrita por dois ou três autores $(70,15 \%)$, e apenas uma pequena fração dos artigos é escrita por mais de cinco autores $(1,28 \%)$, como pode ser verificado na Tabela 1.

Tabela 1 - Número de autores por artigo

\begin{tabular}{c|c|c}
\hline NÚMERO DEAUTORES & ARTIGOS & \% DE ARTIGOS \\
\hline $\mathbf{1}$ & 23 & 3,69 \\
\hline $\mathbf{2}$ & 209 & 33,55 \\
\hline $\mathbf{3}$ & 228 & 36,60 \\
\hline $\mathbf{4}$ & 114 & 18,30 \\
\hline $\mathbf{5}$ & 41 & 6,58 \\
\hline $\mathbf{6}$ & 4 & 0,64 \\
\hline $\mathbf{7}$ & 4 & 0,64 \\
\hline TOTAL & $\mathbf{6 2 3}$ & $\mathbf{1 0 0}$ \\
\hline
\end{tabular}

Fonte: Dados da pesquisa.

Com intuito de investigar os autores mais prolíferos, foi considerado o ordenamento dos autores na listagem de autoria dos artigos. A Tabela 2 apresenta a relação dos autores que tiveram seus nomes mencionados em primeiro lugar nas listagens de autoria dos artigos em ordem decrescente de número de menções. Entre eles, nas primeiras posições, destacam-se: Leonardo Ensslin, com 17 menções; Carlos Francisco Simões Gomes, com 12 menções; Luís Alberto Duncan Rangel, com 10 menções; Luiz Flavio Autran Monteiro Gomes e Sandra 
Ensslin, com 8 menções; Ademar Dutra, André Luís Policani Freitas, André Andrade Longaray e Yuri Gama Lopes, com 7 menções; Renan Felinto de Farias Aires, José Fabiano da Serra Costa e Miguel Afonso Sellito, com 6 menções; e por fim, Helder Gomes Costa e Cristiano Souza Marins, com 5 menções. A Tabela 2 apresenta autores que tiveram, no mínimo, cinco artigos cujos nomes foram mencionados como primeiro autor.

Tabela 2 - Autores mencionados como primeiro autor

\begin{tabular}{l|c}
\hline AUTOR & $\mathbf{N}^{\mathbf{0}}$ \\
\hline Ensslin, Leonardo & 17 \\
\hline Gomes, Carlos Francisco Simões & 12 \\
\hline Rangel, Luís Alberto Duncan & 10 \\
\hline Ensslin, Sandra & 08 \\
\hline Gomes, Luiz Flávio Autran Monteiro & 08 \\
\hline Dutra, Ademar & 07 \\
\hline Freitas, André Luís Policani & 07 \\
\hline Longaray, André Andrade & 07 \\
\hline Lopes, Yuri Gama & 07 \\
\hline Aires, Renan Felinto de Farias & 06 \\
\hline Costa, José Fabiano da Serra & 06 \\
\hline Sellitto, Miguel Afonso & 06 \\
\hline Costa, Helder Gomes & 05 \\
\hline Marins, Cristiano Souza & 05 \\
\hline Fonte: Dados da pesquisa & \\
\hline
\end{tabular}

Fonte: Dados da pesquisa.

A configuração de autores, entretanto, apresenta diferenças importantes se considerada apenas a participação como coautor nos artigos, independe se primeiro autor ou não. Assim, a Tabela 3 apresenta os autores com seus respectivos números de participações com, no mínimo, 10 artigos, independente da ordem de menção dos nomes dos autores. Entre as primeiras posições, destacam-se: Leonardo Ensslin, com 63 participações; Adiel Teixeira de Almeida, com 45 participações; Sandra Ensslin com 42 participações; Helder Gomes da Costa, com 31 participações; Ademar Dutra, com 30 participações; Luiz Flávio Autran Monteiro, com 29 participações; Danielle Costa Morais, com 23 participações; e Valério Antonio Pamplona Salomon, com 21 participações.

Tabela 3 - Autores com mais participações nos artigos selecionados

\begin{tabular}{l|c}
\hline AUTOR & $\mathbf{N}^{\mathbf{0}}$ \\
\hline Ensslin, Leonardo & 63 \\
\hline Almeida, Adiel Teixeira de & 45 \\
\hline Ensslin, Sandra Rolim & 42 \\
\hline Costa, Helder Gomes & 31 \\
\hline Dutra, Ademar & 30 \\
\hline Gomes, Luiz Flávio Autran Monteiro & 29 \\
\hline Morais, Danielle Costa & 23 \\
\hline Salomon, Valério Antonio Pamplona & 21 \\
\hline Costa, Ana Paula Cabral Seixas & 19 \\
\hline Gomes, Carlos Francisco Simões & 19 \\
\hline Alencar, Luciana Hazin & 18 \\
\hline Cavalcante, Cristiano Alexandre Virgínio & 18 \\
\hline Rangel, Luís Alberto Duncan & 18 \\
\hline Freitas, André Luís Policani & 16 \\
\hline Mota, Caroline Maria de Miranda & 14 \\
\hline
\end{tabular}




\begin{tabular}{l|c}
\hline \multicolumn{1}{|c}{} \\
\hline Mello, João Carlos Correia Baptista Soares de & 14 \\
\hline Marins, Fernando Augusto Silva & 13 \\
\hline Sellitto, Miguel Afonso & 13 \\
\hline Almeida Filho, Adiel Teixeira de & 12 \\
\hline Chaves, Maria Cecília de Carvalho & 12 \\
\hline Belderrain, Mischel Carmen Neyra & 11 \\
\hline Pinheiro, Plácido Rogério & 10 \\
\hline
\end{tabular}

Fonte: Dados da pesquisa.

Concluindo o estudo da autoria, verificou-se que as universidades que mais contribuíram para o desenvolvimento do assunto foram determinadas com base nas entidades de filiação dos autores mencionadas nos artigos. A contabilização dos resultados foi realizada por meio do somatório das menções ponderadas pela razão inversa do número de autores dos artigos de origem para cada uma das instituições. Ao todo, foram identificadas 111 instituições, destacando-se a UFPE, com $27,32 \%$ de participação; a UFF, com $10,92 \%$ de participação; a UFSC, com 11,24\% de participação; a UNESP, com 4,41\% de participação; e o IBMEC/RJ, com 4,17\% de participação. A evidenciação dos autores e instituições contribui para identificar os autores e universidades que estão na frente da pesquisa do tema, auxiliando, em especial, os iniciantes a identificar as referências em pesquisa na área.

\subsection{Estudo do conteúdo}

Com relação ao conteúdo dos artigos selecionados, verificaram-se quais são os métodos multicritério de decisão mais aplicados nos últimos 12 anos e em quais áreas são aplicados. O método AHP foi o mais aplicado (201 aplicações), seguido dos métodos MACBETH (137 aplicações), PROMETHEE II (67 aplicações), ELECTRE TRI (41 aplicações) e MAUT (29 aplicações), conforme mostra a Tabela 4 que resume a análise quanto à aplicação dos métodos, revelando a frequência de sua ocorrência no período de estudo (de 2004 a 2016).

Tabela 4 - Resumo das aplicações de métodos multicritério nos artigos selecionados

\begin{tabular}{|c|c|c|c|c|c|c|c|c|c|c|c|c|c|c|c|}
\hline $\begin{array}{l}\text { ANO } \\
\text { MÉTODO }\end{array}$ & 04 & 05 & 06 & 07 & 08 & 09 & 10 & 11 & 12 & 13 & 14 & 15 & 16 & $\mathbf{T}$ & $\%$ \\
\hline AHP & 12 & 12 & 12 & 12 & 10 & 17 & 25 & 15 & 20 & 21 & 23 & 19 & 03 & 201 & 32,26 \\
\hline MACBETH & 11 & 7 & 5 & 11 & 13 & 7 & 9 & 16 & 11 & 13 & 14 & 16 & 04 & 137 & 21,99 \\
\hline PROMETHEE II & 3 & 3 & 5 & 5 & 6 & 5 & 3 & 4 & 3 & 6 & 14 & 7 & 03 & 67 & 10,75 \\
\hline ELECTRE TRI & 5 & 3 & 7 & 6 & 1 & 3 & 2 & 2 & 5 & 4 & 1 & 1 & 01 & 41 & 6,58 \\
\hline MAUT & 3 & 4 & 0 & 1 & 0 & 4 & 4 & 2 & 3 & 3 & 1 & 3 & 01 & 29 & 4,65 \\
\hline TODIM & 0 & 1 & 1 & 3 & 4 & 3 & 3 & 3 & 2 & 1 & 2 & 1 & 0 & 24 & 3,85 \\
\hline ELECTRE II & 3 & 0 & 1 & 1 & 0 & 1 & 1 & 2 & 1 & 6 & 3 & 2 & 0 & 21 & 3,37 \\
\hline THOR & 3 & 2 & 1 & 1 & 2 & 1 & 1 & 0 & 1 & 1 & 0 & 3 & 0 & 16 & 2,57 \\
\hline VIP Analysis & 2 & 4 & 1 & 1 & 2 & 0 & 1 & 3 & 0 & 1 & 0 & 1 & 0 & 16 & 2,57 \\
\hline ELECTRE III & 2 & 0 & 0 & 0 & 1 & 1 & 1 & 2 & 3 & 1 & 3 & 0 & 0 & 14 & 2,25 \\
\hline $\begin{array}{l}\text { SMARTER } \\
\end{array}$ & 0 & 0 & 0 & 0 & 2 & 0 & 1 & 2 & 1 & 1 & 2 & 4 & 0 & 13 & 2,09 \\
\hline ELECTRE I & 2 & 1 & 0 & 1 & 0 & 0 & 3 & 1 & 0 & 1 & 2 & 1 & 0 & 12 & 1,93 \\
\hline $\begin{array}{l}\text { PROMETHEE I } \\
\end{array}$ & 0 & 0 & 0 & 0 & 1 & 2 & 1 & 0 & 3 & 0 & 3 & 1 & 0 & 11 & 1,77 \\
\hline$\overline{\mathrm{ANP}}$ & 0 & 0 & 0 & 0 & 1 & 4 & 0 & 1 & 0 & 0 & 2 & 2 & 01 & 11 & 1,77 \\
\hline ELECTRE IV & 1 & 0 & 0 & 0 & 3 & 2 & 2 & 1 & 0 & 1 & 0 & 0 & 0 & 10 & 1,61 \\
\hline
\end{tabular}

Fonte: Dados da pesquisa. 
Dos 623 artigos analisados, em 83,5\% destes, em sua realização foi aplicado apenas um método multicritério, representando 520 artigos. Quanto às pesquisas com mais de um método de decisão aplicado, representados pelos 16,5\% restantes da análise, observou-se a ocorrência do emprego de dois métodos, três métodos e seis métodos, dos quais 8,7\% correspondem à utilização de dois métodos, totalizando 54 artigos, destacando-se as combinações utilizadas nos trabalhos entre AHP e ANP, entre dois tipos do método ELECTRE e entre dois tipos do método PROMETHEE. Já em $0,6 \%$, foram empregados três métodos multicritério, sendo totalizados apenas quatro artigos e, por fim, um único trabalho foi realizado por meio de uma comitiva de seis métodos, sendo estes o THOR (S1, S2 e S3), o ELECTRE (I e II) e o PROMETHEE II.

A identificação dos métodos multicritério mais empregados sinaliza um direcionamento em termos de pesquisa e de prática gerencial. Em se tratando de pesquisa, é possível sinalizar as preferências de emprego dos métodos e as respectivas abordagens metodológicas e temáticas. No que tange à prática gerencial, a identificação dos métodos multicritérios mais empregados serve como guia para decisão de qual método se mostra mais adequado, dada a contingência que permeia a respectiva tomada de decisão.

Ao analisar as áreas de maior aplicabilidade dos métodos multicritério, tomou-se como base os segmentos econômicos (áreas) nas quais esses métodos foram empregados. Assim, os artigos selecionados no Portfólio Bibliográfico foram classificados de acordo com suas áreas de aplicação. Foram identificados 70 segmentos econômicos. Restringiu-se a análise às áreas com um número maior que 15 artigos. A Tabela 5 apresenta a distribuição das áreas, o número de artigos e a relação percentual desses na amostra.

Tabela 5 - Número de artigos por área

\begin{tabular}{|c|c|c|}
\hline ÁREA & ARTIGOS & $\%$ DE ARTIGOS \\
\hline Tecnologia da Informação/Sistemas de Informação & 36 & 5,78 \\
\hline Gestão Pública & 33 & 5,30 \\
\hline Avaliação/Seleção de Fornecedores & 32 & 5,14 \\
\hline Gestão Estratégica & 29 & 4,65 \\
\hline Localização & 22 & 3,53 \\
\hline Qualidade & 21 & 3,37 \\
\hline Educação & 20 & 3,21 \\
\hline Gestão de Riscos & 19 & 3,05 \\
\hline Manutenção & 19 & 3,05 \\
\hline Sustentabilidade & 19 & 3,05 \\
\hline Gestão Organizacional & 18 & 2,89 \\
\hline Logística & 18 & 2,89 \\
\hline Priorização/Seleção de Projetos & 18 & 2,89 \\
\hline Gestão do Conhecimento & 15 & 2,41 \\
\hline
\end{tabular}

Fonte: Dados da pesquisa.

Como pode ser observado, as cinco áreas de maior aplicabilidade são: Tecnologia/Sistemas de Informação; Gestão Pública; Avaliação/Gestão de Fornecedores; Gestão Estratégica; e Localização. De forma geral, percebe-se que os métodos multicritério são empregados em diversas áreas, mas essas cinco já direcionam uma tendência de aplicabilidade em pesquisa. Em especial, destaca-se o uso dos métodos multicritério em 
Gestão Pública, enfatizando a importância de pesquisas e desenvolvimentos gerenciais nessa área.

\subsection{Estudo das referências bibliográficas}

Ao analisar as referências bibliográficas dos 623 artigos selecionados, buscou-se identificar as obras de maior repercussão na área. Para isso, foram analisadas as 10.037 referências dos artigos do Portfólio Bibliográfico. Tomou-se como ponto de corte o número de 25 citações por obra. O Quadro 2 apresenta as obras de maior repercussão, destacando os autores, o nome e o tipo da obra.

Quadro 2 - Obras de maior repercussão entre os artigos selecionados

\begin{tabular}{|l|l|l|l|}
\hline CITAÇ̃̃ES & AUTORES & TíTULO & TIPO \\
\hline 121 & $\begin{array}{l}\text { GOMES, L. F. A. M.; GOMES, } \\
\text { C. F. S.; ALMEIDA, A. T. }\end{array}$ & $\begin{array}{l}\text { Tomada de Decisão Gerencial: O Enfoque } \\
\text { Multicritério. }\end{array}$ & Livro \\
\hline 117 & $\begin{array}{l}\text { ENSSLIN, L.; MONTIBELLER } \\
\text { NETO, G.; NORONHA, S. M. }\end{array}$ & $\begin{array}{l}\text { Apoio à Decisão: Metodologia para Estruturação de } \\
\text { Problemas e Avaliação Multicritério de Alternativas. }\end{array}$ & Livro \\
\hline 91 & $\begin{array}{l}\text { GOMES, L. F. A. M.; ARAYA, } \\
\text { M. C. G.; CARIGNANO, C. }\end{array}$ & $\begin{array}{l}\text { Tomada de Decisões em Cenários Complexos: } \\
\text { Introdução aos Métodos Discretos do Apoio } \\
\text { Multicritério à Decisão. }\end{array}$ & Livro \\
\hline 83 & KEENEY, R. L.; RAIFFA, H. & $\begin{array}{l}\text { Decision with Multiple Objectives: Preferences and } \\
\text { Value Tradeoffs. }\end{array}$ & Livro \\
\hline 69 & SAATY, T. L. & Método de Análise Hierárquica. & Livro \\
\hline 69 & VINCKE, P. & Multicriteria Decision-Aid. & Livro \\
\hline 60 & BELTON, V.; STEWART, T. J. & $\begin{array}{l}\text { Multiple Criteria Decision Analysis: An Integrated } \\
\text { Approach. }\end{array}$ & Livro \\
\hline 54 & KEENEY, R. L. & $\begin{array}{l}\text { Value Focused-Thinking: A Path to Creative } \\
\text { Decision-Making. }\end{array}$ & Livro \\
\hline 53 & ROY, B. & Multicriteria Methodology for Decision Aiding. & Livro \\
\hline 47 & ROY, B.; BOUYSSOU, D. & Aide Multicritère à la Décision: Méthodes et Cas. & Livro \\
\hline 47 & SAATY, T. L. & The Analytic Hierarchy Process. & Livro \\
\hline 37 & $\begin{array}{l}\text { ALMEIDA, A. T.; COSTA, A. P. } \\
\text { C. S. }\end{array}$ & $\begin{array}{l}\text { Aplicações com Métodos Multicritério de Apoio à à } \\
\text { Decisão. }\end{array}$ & Livro \\
\hline 34 & ROY, B.; BOYSSOU, D. & Mèthodologie Multicritère d'Aide à la Dècision. & Livro \\
\hline 31 & GOODWIN, P.; WRIGHT, G. & Decision Analysis for Management Judgment. & Livro \\
\hline 29 & ALMEIDA, A. T. & $\begin{array}{l}\text { O Conhecimento e o Uso de Métodos Multicritério } \\
\text { de Apoio à Decisão. }\end{array}$ & Livro \\
\hline 29 & LANDRY, M. & A note on the Concept of Problem. & Artigo \\
\hline 27 & $\begin{array}{l}\text { BANA E COSTA, C. A.; } \\
\text { ENSSLIN, L.; CORRÊA, E. C.; } \\
\text { VANSNICK, J. C. }\end{array}$ & $\begin{array}{l}\text { Decision Support Systems in Action: Integrated } \\
\text { Application in a Multicriteria Decision aid Process. }\end{array}$ & Artigo \\
\hline 26 & ROY, B. & Decision Science or Decision-Aid Science? & Artigo \\
\hline
\end{tabular}

Fonte: Dados da pesquisa.

Ao analisar o Quadro 2, é possível destacar a importância que os livros da área têm nos artigos publicados. Ao total, das 18 referências mais citadas, 15 são livros. Ademais, o Quadro 2 auxilia na identificação das obras de referência, com destaque para as duas primeiras listadas, com mais de 100 citações cada.

Após verificar, dentre os artigos selecionados, quais os de maior repercussão, prossegue-se e finaliza-se o levantamento bibliométrico, analisando aqueles de maior relevância. Para isso, observou-se o somatório de dois indicadores: 1) número de citações no 
site Google Acadêmico; 2) número de citações nas próprias referências bibliográficas dos artigos selecionados.

O site Google Acadêmico traz, para cada artigo pesquisado, o número de vezes que o mesmo foi citado em outros documentos. Sob essa perspectiva, cada um dos 623 artigos selecionados foi pesquisado, no site, no dia 8 de julho de 2016, para a formação do primeiro indicador. O segundo indicador, por sua vez, foi formado por meio da contagem das citações dos artigos selecionados nas mesmas 10.037 referências utilizadas para a determinação das obras de maior repercussão.

Indica-se, na Tabela 6, a lista dos 15 artigos de maior relevância. A primeira coluna da Tabela apresenta o título do artigo; a segunda, o ano de publicação; a terceira, a fonte do artigo; a quarta coluna, o número de citações no Google Acadêmico "GA"; a quinta, o número de citações nas referências bibliográficas "RB".

Tabela 6 - Artigos selecionados em ordem decrescente de relevância

\begin{tabular}{|c|c|c|c|c|}
\hline TÍTULO & ANO & FONTE & G A & RB \\
\hline $\begin{array}{l}\text { Avaliação do desempenho de empresas terceirizadas com o } \\
\text { uso da metodologia Multicritério de Apoio à Decisão- } \\
\text { Construtivista. }\end{array}$ & 2010 & Pesquisa Operacional & 68 & 27 \\
\hline $\begin{array}{l}\text { Visão multicritério da avaliação de programas de pós- } \\
\text { graduação pela Capes: o caso da área engenharia iii baseado } \\
\text { nos métodos ELECTRE II E MAUT. }\end{array}$ & 2004 & Gestão \& Produção & 30 & 17 \\
\hline $\begin{array}{l}\text { Avaliação do tamanho de aeroportos portugueses com } \\
\text { relações multicritério de superação. }\end{array}$ & 2005 & Pesquisa Operacional & 34 & 11 \\
\hline $\begin{array}{l}\text { Modelo multicritério de apoio à decisão para o planejamento } \\
\text { de manutenção preventiva utilizando PROMETHEE II em } \\
\text { situações de incerteza. }\end{array}$ & 2005 & Pesquisa Operacional & 25 & 13 \\
\hline $\begin{array}{l}\text { Medição de desempenho ambiental baseada em método } \\
\text { multicriterial de apoio à decisão: estudo de caso na indústria } \\
\text { automotiva. }\end{array}$ & 2006 & Gestão \& Produção & 34 & 3 \\
\hline $\begin{array}{l}\text { Análise de decisão multicritério na localização de usinas } \\
\text { termoelétricas utilizando sig. }\end{array}$ & 2005 & Pesquisa Operacional & 28 & 7 \\
\hline $\begin{array}{l}\text { Using MCDA methods THOR in an application for } \\
\text { outranking the ballast water management options. }\end{array}$ & 2005 & Pesquisa Operacional & 22 & 12 \\
\hline $\begin{array}{l}\text { Avaliação comparativa do desempenho de três cadeias de } \\
\text { suprimentos em manufatura. }\end{array}$ & 2006 & Produção & 24 & 3 \\
\hline $\begin{array}{l}\text { Avaliação do desempenho de uma manufatura de } \\
\text { equipamentos eletrônicos segundo critérios de competição. }\end{array}$ & 2006 & Produção & 22 & 4 \\
\hline $\begin{array}{l}\text { Multicriteria decision group model for the selection of } \\
\text { suppliers. }\end{array}$ & 2008 & Pesquisa Operacional & 18 & 8 \\
\hline $\begin{array}{l}\text { ELECTRE TRI aplicado à avaliação da satisfação de } \\
\text { consumidores. }\end{array}$ & 2007 & Produção & 17 & 8 \\
\hline $\begin{array}{l}\text { Seleção dos objetivos fundamentais de uma rede de } \\
\text { cooperação empresarial. }\end{array}$ & 2004 & Gestão \& Produção & 22 & 2 \\
\hline $\begin{array}{l}\text { Estruturação de um índice consolidado de desempenho } \\
\text { utilizando o AHP. }\end{array}$ & 2007 & Gestão \& Produção & 20 & 4 \\
\hline 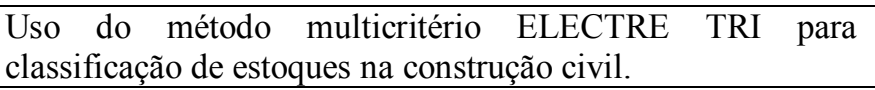 & 2006 & Pesquisa Operacional & 17 & 7 \\
\hline $\begin{array}{l}\text { Análise do Relacionamento entre a Contabilidade Gerencial } \\
\text { e o Processo de Planejamento das Organizações Brasileiras. }\end{array}$ & 2007 & $\mathrm{RAC}$ & 22 & 2 \\
\hline
\end{tabular}

Fonte: Dados da pesquisa.

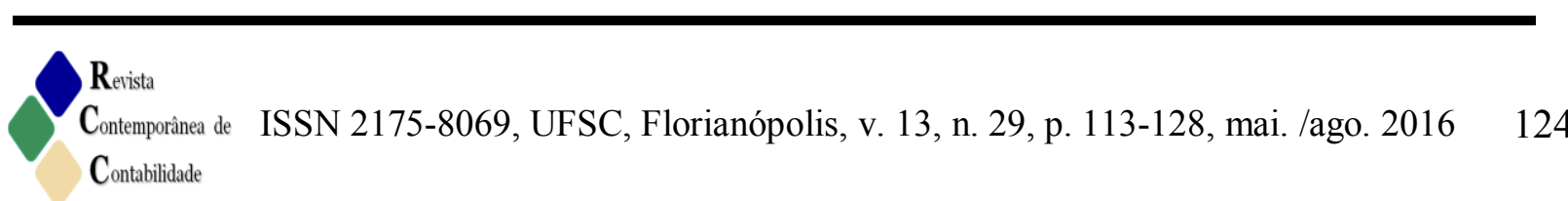


Verificou-se, ainda, que, dos 623 artigos selecionados no Portfólio Bibliográfico, 436 não possuem nenhuma citação. Dos 187 artigos remanescentes com citações, cerca de 11,2\% deles (21 artigos) são responsáveis por mais de 50\% das citações.

Deve-se levar em consideração, na análise precedida, que um número significativo de artigos foi publicado a partir de 2011, o que explica, em parte, o baixo percentual de artigos da amostra que possuem citações.

Da mesma forma, os autores mais citados são, em quase sua totalidade, pesquisadores com grande experiência acadêmica, orientadores de mestrado e doutorado, que possuem tradição e reconhecimento de suas pesquisas na área de métodos multicritério em âmbito nacional.

Em uma perspectiva qualitativa, a análise permite inferir que ainda há métodos e segmentos de estudo pouco explorados. No exame dos artigos, um número pequeno de pesquisas foram identificadas, por exemplo, de aplicação de métodos multicritério para apoio à decisão gerencial na área da saúde. Paralelamente, alguns dos métodos multicritério elencados neste artigo (ver Tabela 4) são mais utilizados em pesquisas no Brasil (AHP e MACBETH), em detrimento de outros (TODIM, ELECTRE, VIPanalysis).

\section{Considerações Finais}

Este estudo teve como objetivo a caracterização da produção científica brasileira sobre a aplicação de métodos multicritério de apoio à decisão, publicada no período compreendido entre 2004 e 2016, por meio de uma análise de artigos disponíveis em bases de dados online. Para isso, adotou-se a perspectiva da bibliometria na realização do estudo da produção, da autoria, do conteúdo e das referências bibliográficas de artigos selecionados de anais e periódicos de destaque nacional sobre o tema da pesquisa.

Entre os principais resultados encontrados na análise bibliométrica, constatou-se uma tendência crescente de artigos por ano com a temática estudada, dos quais a maioria é escrita por dois ou três autores, sendo aqueles que se destacam na área: Leonardo Ensslin, Carlos Francisco Simões Gomes, Luís Alberto Duncan Rangel, Luiz Flavio Autran Monteiro Gomes e Sandra Ensslin. Já as universidades que mais se destacaram em termos de produção científica, em ordem decrescente, foram: UFPE, UFF e UFSC. Esses resultados assinalam os autores e universidades que podem ser considerados como referência na área, auxiliando tanto os que visam iniciar com pesquisa na área, quanto os que desejam se aprofundar, como os profissionais da gestão que desejam referências para soluções de seu campo de atuação.

Com relação aos métodos multicritério mais aplicados, o AHP é o mais utilizado, seguido dos métodos ELECTRE, MACBETH, PROMETHEE e MAUT. Esse achado da pesquisa contribui para evidenciar as preferências em termos de métodos mais aplicados, contribuindo para o direcionamento de novas pesquisas e soluções em termos de gestão.

Cabe também destacar as áreas de atuação em que serviram de contexto para as pesquisas em métodos multicritério. Em especial, é relevante destacar a capilaridade de aplicação dos métodos, bem como a área de gestão pública. Recomenda-se a continuidade e ampliação das pesquisas com métodos multicritério nessa área, contribuindo para sedimentação de uma base de conhecimento científico que permita a integração com a prática.

A pesquisa encontrou como limitação a impossibilidade de analisar a totalidade da produção científica brasileira, em função do elevado número de artigos publicados nas mais variadas fontes. O número de referências bibliográficas dos artigos selecionados, que supera a 
casa dos milhares, revelou-se o item de maior dificuldade na análise, devido ao grande volume de dados e à falta de padronização destes.

Como recomendações para futuras pesquisas, sugere-se a ampliação do estudo com a inclusão de periódicos e congressos internacionais. Outra possibilidade é o estudo da aplicação dos métodos multicritérios de apoio à decisão em outras áreas do conhecimento, além da produção, gestão e das ciências contábeis, como exemplo: gestão das organizações sociais, operações e logística sustentáveis e operações humanitárias.

\section{Referências}

ALBUQUERQUE, Janison Machado. Avaliação dos controles internos de uma instituição pública de ensino superior à luz da metodologia MCDA-construtivista

Revista Contemporânea de Contabilidade, Florianópolis, v. 8, n. 15, p. 129-150, 2011.

ARAÚJO, Carlos Alberto. Bibliometria: evolução histórica e questões atuais. Em Questão, Porto Alegre, v. 12, n. 1, p. 11-32, jan./jun. 2006.

BANA E COSTA, Carlos Antonio, VANSNICK, Jean-Claude. A new approach to the problem of building a value function: MACBETH. Investigação Operacional, 15:15-35, 1995.

BANA E COSTA, Carlos. Antonio; DE CORTE, Jean Marie, VANSNICK, Jean- Claude. On the mathematical foundations of Macbeth. In: Greco, J., Ehrgott, S. (Ed.) Multicriteria Decision Analysis: state of the art survey. Boston: Springer Verlag, p. 409-442, 2005.

BARBA-ROMERO, Sergio, POMEROL, Jean-Charles. Decisiones multicriterio: fundamentos teóricos y utilización práctica. España: Universidad de Alcalá, 1997. p. 420.

BELTON Valerie, STEWART Theodore. Multiple criteria decision analysis: An integrated approach. Boston: Kluwer Academic Publishers, 2002.

BORTOLUZZI, S. C.; SILVA, M. R.; ENSSLIN, S. R.; ENSSLIN, L. Estruturação de um modelo de Avaliação de Desempenho para a gestão do Curso de Ciências Contábeis da Universidade Tecnológica Federal do Paraná. Revista de Educação e Pesquisa em Contabilidade, v. 7, n. 1, p. 35-57, 2013.

BRANS, Jean Pierre. L'ingénierie de la décision: élaboration d'instruments d'aide à la décision. La méthode PROMETHEE. Presses de l’Université Laval, 1982.

BRANS, Jean. Pierre, VINCKE, Philippe, MARESCHAL, Bertrand. How to select and how to rank projects: the promethee methods. European Journal of Operational Research, North Holland, v. 24, n. 2, p. 228-238, 1986.

DUTRA, A.; RIPOLL-FELIU, V. M.; FILLOL, A. G.; ENSSLIN, S. R.; ENSSLIN, L. The construction of knowledge from the scientific literature about the theme seaport performance 
evaluation. International Journal of Productivity and Performance Management, v. 64, n. 2, p. 243-269, 2015.

ENSSLIN, Leonardo, MONTIBELLER, Gilberto, NORONHA Sandro, MacDonald. Apoio à Decisão: Metodologias para Estruturação de Problemas e Avaliação Multicritério de Alternativas. Florianópolis: Insular, 2001.

ENSSLIN, S. R.; ENSSLIN, L.; IMLAU, J. M.; CHAVES, L. C. Processo de mapeamento das publicações científicas de um tema: portfólio bibliográfico e análise bibliométrica sobre avaliação de desempenho de cooperativas de produção agropecuária. Revista de Economia e Sociologia Rural (Impresso), v. 52, p. 587-608, 2014.

ENSSLIN, L.; DUTRA, A.; MARTINS, R. P.; DEZEM, V. Modelo Construtivista para Apoiar o Processo de Gestão da Universidade Federal de Tocantins. Revista IberoAmericana de Estratégia, v. 15, n. 2, p. 122-129, 2016.

GIL, Antonio Carlos. Como elaborar projetos de pesquisa. 5. ed. São Paulo: Atlas, 2010.

KEENEY, Ralph, RAIFFA, Howard. Decisions with multiple objectives: preferences and value trade-offs. Wyley, New York, 1976.

HWANG, Chig-Lai, LAI, Youn Jou, LIU, Tseung. A new approach for multiple objective decision making. Computers and Operational Research, 20:889-99, 1993.

LONGARAY, André Andrade. A Teoria da Decisão Revisitada: Análise e Perspectivas. Revista ANGRAD - Associação Nacional dos Cursos de Graduação em Administração, Rio de Janeiro, v. 8, n. 2, p. 137-151, abr./maio/jun. 2007.

LONGARAY, A. A.; POPIOLEK Jr., T. L.; MUNHOZ, P. R.; GERI, F. S.; CASTELLI, T. M. Caracterização da produção científica brasileira sobre a aplicação de métodos multicritério de apoio à decisão: uma análise das publicações entre 2004-2013. In: XXXV Encontro

Nacional de Engenharia de Produção, 2015.

RANGEL, Luís Alberto Duncan; GOMES, Luiz Flávio Autran Monteiro. O Apoio Multicritério à Decisão na avaliação de candidatos. Produção, São Paulo, v. 20, n. 1, p. 92101, mar. 2010.

RODRIGUEZ, Dey Salvador Sanchez; COSTA, Helder Gomes; CARMO, Luiz Felipe Roris Rodriguez Scavarda do. Multicriteria decision aid methods applied to PPC problems: a mapping of papers published in Brazilian journals. Gestão \& Produção, São Carlos, v. 20, n. 1, p. 134-146, jan./mar. 2013.

ROESCH, Sylvia Maria Azevedo. Projetos de estágio e de pesquisa em Administração: guia para estágios, trabalhos de conclusão, dissertações e estudos de caso. 3. ed. São Paulo: Atlas, 2013. 
ROMERO, Carlos. Teoría de la decisión multicriterio: conceptos, técnicas y aplicaciones. Madrid: Alianza, 1993. p. 195.

ROY, Bernard. The outranking approach and the foundations of electre methods. Theory and Decision, Netherlands, v. 31, p. 49-73, 1991.

ROY, Bernard, BOUYSSOU, Denis. Aide multicritère à la décision: méthodes et cas. Paris: Econômica, 1993. p. 695.

SAATY, Thomas. How to make a decision: the analytic hierarchy process. European Journal Operations Research, v.48, n.1, p.9-26, 1990.

SCHÄRLIG, Alain. Pratiquer electre et prométhée: un complément à décider sur plusieurs critères. Lausanne: Press Polytechniques e Universitaires Romande, 1996. p. 173.

TRIANTAPHYLLOU, Evangelos, SHU, B., NIETO SANCHEZ, Salvador, RAY, Toni. Multi-Criteria Decision Making: an Operations Research Approach. Encyclopedia of Electrical and Electronics Engineering, 15, p. 175-186, 1998.

VALMORBIDA, S. M. I.; ENSSLIN, L. Construção de conhecimento sobre avaliação de desempenho para gestão organizacional: uma investigação nas pesquisas científicas internacionais. Revista Contemporânea de Contabilidade, Florianópolis, v. 13, n. 28, p. 123-148, 2016.

VINCKE, Philippe. L'aide multicritère à la dècision. Bruxelles: Éditions de 1'Université de Bruxelles, 1989. p. 179. 